\title{
Participant Object
}

National Cancer Institute

\section{Source}

National Cancer Institute. Participant Object. NCI Thesaurus. Code C79269.

Something that takes part in an activity. 